\title{
AN OPTIMIZED FAST VOLTAGE STABILITY INDICATOR
}

\author{
C. A. Belhadj \\ M. A. Abido
}

Electrical Engineering Department

King Fahd University of Petroleum \& Minerals

Dhahran 31261, Saudi Arabia

\begin{abstract}
This paper proposes a simulated annealing optimization technique for optimal voltage stability profile through out the whole power network. The technique is applied to control the power elements of major influence on the voltage stability profile. Elements such as generator reactive generation, adjustable shunt compensation devices, transformer tap settings are optimally adjusted at each operating point to reach the objective of minimizing the voltage stability index at each individual bus as well minimizing the global voltage stability indicator. Because of the optimal setting of the control elements, the maximum possible MVA voltage stable loading has been achieved and a the best voltage profile was obtained. Results of tests conducted on the 6 bus Wale and Hale system are presented and discussed.
\end{abstract}

Keywords: Optimization Technique, Simulated Annealing, Voltage Stability Monitoring, Indicator and Margin.

\section{INTRODUCTION}

The power system ability to maintain constantly acceptable bus voltage at each node under normal operating conditions, after load increase, following system configuration changes or when the system is being subjected to a disturbance is a very important characteristic of the system. The non-optimized control of VAR resources may lead to progressive and uncontrollable drop in voltage resulting in an eventual wide spread voltage collapse.

The phenomenon of voltage instability is attributed to the power system operation at its maximum transmissible power limit, shortage of reactive power resources and inadequacy of reactive power compensation tools. A non optimized setting of the level or control of the reactive resources play an effective role to expedite the voltage unstability and to speed up reaching the maximum loading limit.

The main factors contributing to the voltage collapse are the generators reactive power limit, voltage control limits, load characteristics, reactive compensation devices characteristics and their actions.

Paper BPT99-363-12 accepted for presentation at the IEEE Power Tech'99 Conference, Budapest, Hungary, Aug 29 - Sep 2, 1999.
Voltage stability estimation techniques based on load flow Jacobian analysis such as, singular value decomposition, Eigenvalue calculations, sensitivity factories, and modal analysis are time consuming for a large power system [1-4].

Several indices based methods such as Voltage Instability Proximity Index (VIPI) and Voltage Collapse Proximity Indicator (VCPI) are used to evaluate voltage instability are. They are based on multiple load flow solutions and give only global picture [5,6]. The transmission proximity index that specifies the weakest transmission part of the system based on voltage phasor approach necessitate the scanning of the whole power system structure for several time which the time consuming approach [7].

The strong tie of the voltage stability problem with the reactive power resources and flow in the system raise the interest in optimizing the rescheduling of the VAR control tools. An optimum VAR picture would maintain a good voltage profile and extend the maximum loading capability of the power network.

Several approaches for optimal reactive power picture have been reported in the literature. Methods such as nonlinear programming and linear programming algorithms were applied. They are complex, time consuming and require considerable amount of memory [10 -14].

The non-incremental quadratic programming model used for optimal reactive power control Though the technique is relatively accurate and show a satisfactory convergence characteristics but as the system gets bigger the number of variables to be evaluated would rise sharply [15].

The present work proposes an optimized fast voltage stability indicator dedicated for evaluation and monitoring. The optimized index gives information covering the whole power system and evaluated at each individual bus, Its is calculated at every operating point. The used indicator is simple to derive and fast to calculate.

In order to enhance the voltage stability profile through out the whole power network, simulated annealing (SA) optimization technique [16-17] is applied to control the power elements of major influence on the voltage stability profile. Elements such as generator reactive generation, adjustable shunt compensation devices, transformer tap settings are optimally adjusted at each 
operating point to reach the objective of increasing the distance from an unstable system state and therefore to increase the maximum possible system safe loading. The objective is achieved through minimizing the L-index values at every bus of the system and consequently the global power system L-index.

\section{FAST VOLTAGE STABILITY INDICATOR}

For voltage stability bus evaluation in connection with transmission path, an indicator L-index is used [9]. The indicator value varies in the range between 0 (the no load case) and 1 which corresponds to

Magnitude and phase along with the power network information provided by the load flow program.

For multi-node system

$$
I_{b u s}=Y_{b u s} \times V_{b u s}
$$

By segregating the load buses (PQ) from generator buses (PV), equation (1) can write as

$$
\begin{aligned}
& {\left[\begin{array}{c}
I_{L} \\
I_{G}
\end{array}\right]=\left[\begin{array}{ll}
Y_{1} & Y_{2} \\
Y_{3} & Y_{4}
\end{array}\right]\left[\begin{array}{l}
V_{L} \\
V_{G}
\end{array}\right]} \\
& {\left[\begin{array}{l}
V_{L} \\
I_{G}
\end{array}\right]=\left[\begin{array}{ll}
H_{1} & H_{2} \\
H_{3} & H_{4}
\end{array}\right]\left[\begin{array}{l}
I_{L} \\
V_{G}
\end{array}\right]}
\end{aligned}
$$

$\mathrm{V}_{\mathrm{L}}, \mathrm{I}_{\mathrm{L}}$ : Voltages and Currents for PQ buses

$\mathrm{V}_{\mathrm{G}}, \mathrm{I}_{\mathrm{G}}$ : Voltages and Currents for PV buses

Where, $\mathrm{H}_{1}, \mathrm{H}_{2}, \mathrm{H}_{3}, \mathrm{H}_{4}$ : submatrices generated from $\mathrm{Y}_{\text {bus }}$ partial inversion.

Let

$$
\bar{V}_{o k}=\sum_{i=1}^{n G} H_{2 k i} \cdot \bar{V}_{i}
$$

$\mathrm{n}_{\mathrm{G}}:$ number of generators

$$
H_{2}=-Y_{1} \times Y_{2}
$$

$$
L_{K}=\left|1+\frac{V_{o k}}{V_{K}}\right|
$$

\section{$\mathrm{L}_{\mathrm{k}}: \mathrm{L}$-index voltage stability indicator for bus $\mathrm{k}[8,9]$}

Stability requires that $\mathrm{L}_{\mathrm{k}}<1$ and must not be violated on a continuous basis. Hence a global system indicator $\mathrm{L}$ describing the stability of the complete system is $\mathrm{L}=\mathrm{L}_{\max }$ $\left\{\mathrm{L}_{\mathrm{k}}\right\}$, where in $\left\{\mathrm{L}_{\mathrm{k}}\right\}$ all $\mathrm{L}$ bus indexes are listed.

In practice $\mathrm{L}_{\max }$ must be lower than a threshold value. The predetermined threshold value is specified at the planning stage depending on the system configuration and on the utility policy regarding the quality of service and the level of system decided allowable margin. In practice, the calculation of the complex vector $\mathrm{V}_{\mathrm{ok}}$ never uses the inversion of $\mathrm{Y}_{1}$.

$$
\left[-Y_{1}\right] \cdot V_{o k}=\left[Y_{2}\right] \cdot V_{G}
$$

Instead sparse factorization vector methods have been used to solve the linear system (7) and make from L-index a potential candidate for real-time performance $[8,9]$

\section{SIMULATED ANNEALING TECHNIQUE}

\subsection{Overview}

Simulated annealing is an optimization technique that simulates the physical annealing process in the field of combinatorial optimization. Annealing is the physical process of heating up a solid until it melts, followed by slow cooling it down by decreasing the temperature of the environment in steps. At each step, the temperature is maintained constant for a period of time sufficient for the solid to reach thermal equilibrium.

Metropolis et al [17] proposed a Monte Carlo method to simulate the process of reaching thermal equilibrium at a fixed value of the temperature $T$. In this method, a randomly generated perturbation of the current configuration of the solid is applied so that a trial configuration is obtained. This trial configuration is accepted and becomes the current configuration if it satisfies an acceptance criterion. The process continues until the thermal equilibrium is achieved after a large number of perturbations. By gradually decreasing the temperature $T$ and repeating Metropolis simulation, new lower energy levels become achievable. As $T$ approaches zero least energy configurations will have a positive probability of occurring.

\subsection{SA Algorithm}

At first, the analogy between a physical annealing process and a combinatorial optimization problem is based on the following [16]:

- Solutions in an optimization problem are equivalent to configurations of a physical system.

- The cost of a solution is equivalent to the energy of a configuration.

In addition, a control parameter $C_{p}$ is introduced to play the role of the temperature $T$.

The basic elements of SA are defined as follows :-

- Current, trial, and best solutions, $x_{\text {current }}, x_{\text {trial }}$, and $x_{\text {best }}$ : these solutions are sets of the optimized parameter values at any iteration.

- Acceptance criterion: at any iteration, the trial solution can be accepted as the current solution if it meets one of the following critera; (a) $J\left(x_{\text {trial }}\right)<$ $J\left(x_{\text {current }}\right)$; (b) $J\left(x_{\text {trial }}\right)>J\left(x_{\text {current }}\right)$ and $\exp \left(-\left(J\left(x_{\text {trial }}\right)-\right.\right.$ $\left.\left.J\left(x_{\text {current }}\right)\right) / \quad C_{p}\right) \geq \operatorname{rand}(0,1)$. Here, $\operatorname{rand}(0,1)$ is a random number with domain $[0,1]$ and $J\left(x_{\text {trial }}\right)$ and $J\left(x_{\text {current }}\right)$ are the objective function values associated with $x_{\text {trial }}$ and $x_{\text {current }}$ respectively. Criterion (b) indicates that the trial solution is not necessarily rejected if its objective function is not as good as that of the current solution with hoping that a much better solution become reachable. 
- Acceptance ratio: at a given value of $C_{p}$, an $n_{1}$ trial solutions can be randomly generated. Based on the acceptance criterion, an $n_{2}$ of these solutions can be accepted. The acceptance ratio is defined as $n_{2} / n_{1}$.

- Cooling schedule: it specifies a set of parameters that governs the convergence of the algorithm. This set includes an initial value of control parameter $C_{p 0}$, a decrement function for decreasing the value of $C_{p}$, and a finite number of iterations or transitions at each value of $C_{p}$, i.e. the length of each homogeneous Markov chain. The initial value of $C_{p}$ should be large enough to allow virtually all transitions to be accepted. However, this can be achieved by starting off at a small value of $C_{p 0}$ and multiplying it with a constant larger than $1, \alpha$, i.e. $C_{p 0}=\alpha C_{p 0}$. This process continues until the acceptance ratio is close to 1 . This is equivalent to heating up process in physical systems. The decrement function for decreasing the value of $C_{p}$ is given by $C_{p}=\mu C_{p}$ where $\mu$ is a constant smaller than but close to 1 . Typical values lie between 0.8 and 0.99 [16].

- Equilibrium condition: it occurs when the current solution does not change for a certain number of iterations at a given value of $C_{p}$. It can be achieved by generating a large number of transitions at that value of $C_{p}$.

- Stopping Criteria: these are the conditions under which the search process will terminate. In this study, the search will terminate if one of the following criteria is satisfied: (a) the number of Markov chains since the last change of the best solution is greater than a prespecified number; or, (b) the number of Markov chains reaches the maximum allowable number.

The SA algorithm can be described in steps as follows:

Step 1: Set the initial value of $C_{p 0}$ and randomly generate an initial solution $x_{\text {initial }}$ and calculate its objective function. Set this solution as the current solution as well as the best solution, i.e. $x_{\text {initial }}=x_{\text {current }}=x_{\text {best }}$.

Step 2: Randomly generate an $n_{1}$ of trial solutions in the neighborhood of the current solution.

Step 3: Check the acceptance criterion of these trial solutions and calculate the acceptance ratio. If acceptance ratio is close to 1 go to step 4 ; else set $C_{p 0}=\alpha C_{p 0}, \alpha>1$, and go back to step 2 .

Step 4: Set the chain counter $k_{c h}=0$.

Step 5: Generate a trial solution $x_{\text {trial }}$. If $x_{\text {trial }}$ satisfies the acceptance criterion set $x_{\text {current }}=x_{\text {trial }}, J\left(x_{\text {current }}\right)=$ $J\left(x_{\text {trial }}\right)$, and go to step 6; else go to step 6.

Step 6: Check the equilibrium condition. If it is satisfied go to step 7; else go to step 5 .

Step 7: Check the stopping criteria. If one of them is satisfied then stop; else set $k_{c h}=k_{c h}+1$ and $C_{p}=\mu C_{p}$, $\mu<1$, and go back to Step 5 .

\section{4}

\section{TEST RESULTS AND DISCUSSION}

The demonstrated Wale and Hale 6 bus test system shown in Fig. 1, which has MVAR limits on generator two buses where one two-controllable transformer taps between upper and lower settings and two variable capacitors. The line data and bus data are given in Tables 1 and 2 respectively. The voltage stability index is evaluated at every operating point and for every bus in the system along the system over all index $\mathrm{L}_{\max }$. The simulated annealing optimization is activated of every operating in order to adjust the available VAR control tools for the objective of minimizing the value of L-index at every bus in the system and consequently the system overall voltage stability indicator $\mathrm{L}_{\max }$. Hence, the optimization problem can be written as

Minimize ( $\max \left\{\mathrm{L}_{\mathrm{k}} ; \mathrm{k}=1,2, \ldots\right.$, number of buses $\left.\}\right)(8)$

The problem constraints are the control variable bounds as given in Table 3 .

The objective function convergence rate is shown in Fig. 2. It can be shown that the fast convergence of the proposed technique. The optimal values of control variables are given in Table 3. Also, Table 3 shows the load flow solution with the initial settings and the proposed optimal settings of the control variables. It is clear that the voltage profile is greatly improved. In addition the real power loss is reduced by $11.3 \%$.

Table 4 shows a comparative list of results using both voltage stability evaluation of L-index with and without optimization. It can be seen that the values of L-index at load buses are reduced, therefore, the voltage stability of the system is enhanced and improved.

The test was carried out for a different load level starting from $40 \%$ of the base load with a step increase of all loads in the system till voltage collapse.

The voltages of load buses versus load factor without and with optimization are shown in Fig. 3. In addition, Lindex values at load buses versus load factor are shown in Fig. 4. It is clear that the application of the SA algorithm has significantly reduced the values of L-index all over the system. Consequently, the voltage stability distance from collapse has increased. The gain in power system MVA loading was found to be $23 \%$. The above both positive results demonstrate the potential of the proposed approach to improve and enhance the system voltage stability.

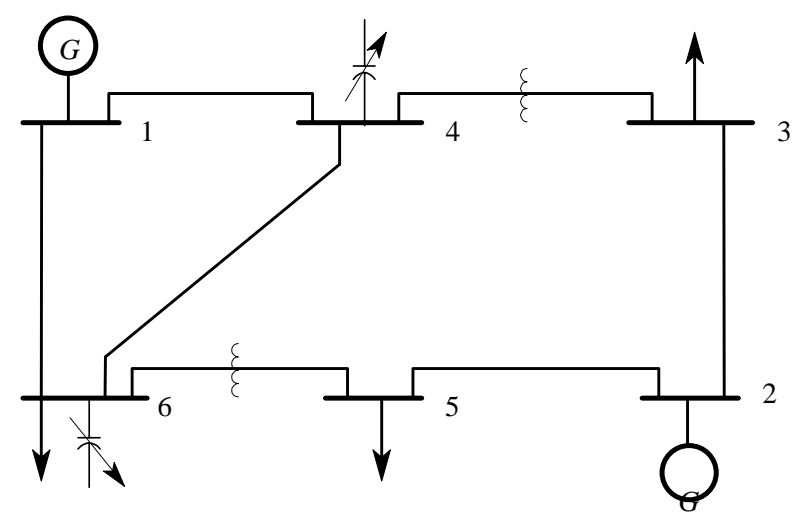

Fig. 1: Single line diagram of the 6-bus system 
TABLE 1: LINE DATA ON 100 MVA BASE

\begin{tabular}{cccccc}
\hline Line\# & From & To & $\mathrm{R}(\mathrm{pu})$ & $\mathrm{X}(\mathrm{pu})$ & Tap Ratio \\
\hline 1 & 1 & 6 & 0.123 & 0.518 & ---- \\
2 & 1 & 4 & 0.080 & 0.370 & ---- \\
3 & 4 & 6 & 0.097 & 0.407 & ---- \\
4 & 6 & 5 & 0.000 & 0.300 & $1.025: 1.0$ \\
5 & 5 & 2 & 0.282 & 0.640 & ---- \\
6 & 2 & 3 & 0.723 & 1.050 & ---- \\
7 & 4 & 3 & 0.000 & 0.133 & $1.100: 1.0$ \\
\hline
\end{tabular}

TABLE 2: BUS DATA ON 100 MVA BASE

\begin{tabular}{ccccc}
\hline Bus\# & $\mathrm{V}(\mathrm{pu})$ & $\mathrm{P}_{\mathrm{g}}(\mathrm{pu})$ & $\mathrm{P}_{\mathrm{L}}(\mathrm{pu})$ & $\mathrm{Q}_{\mathrm{L}}(\mathrm{pu})$ \\
\hline 1 & 1.05 & ---- & ---- & --- \\
2 & 1.10 & 0.50 & 0.00 & 0.00 \\
3 & 1.00 & ---- & 0.55 & 0.13 \\
4 & 1.00 & ---- & 0.00 & 0.00 \\
5 & 1.00 & ---- & 0.30 & 0.18 \\
6 & 1.00 & ---- & 0.50 & 0.05 \\
\hline
\end{tabular}

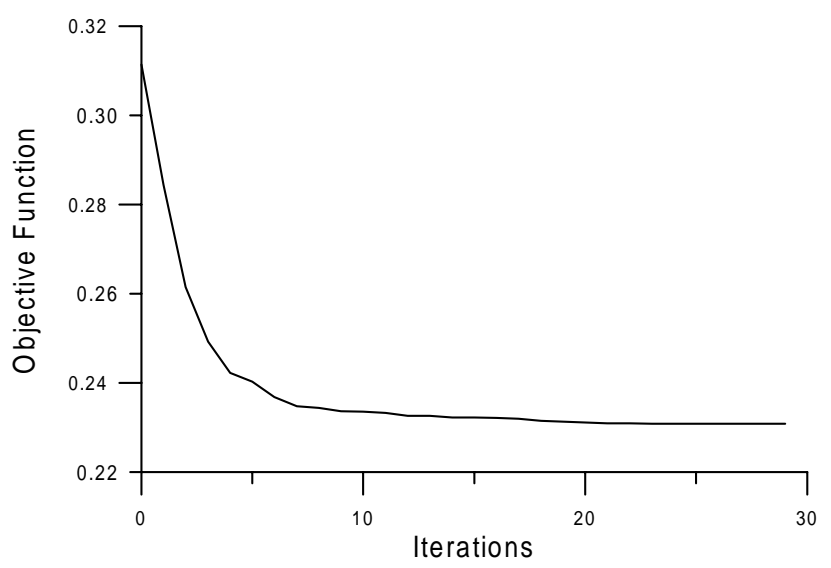

Fig. 2: Convergence of the objective function

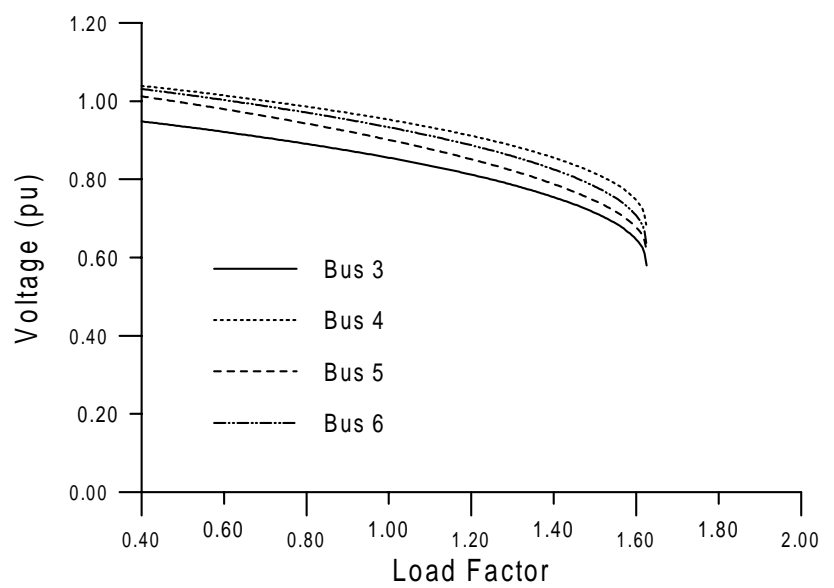

(a)

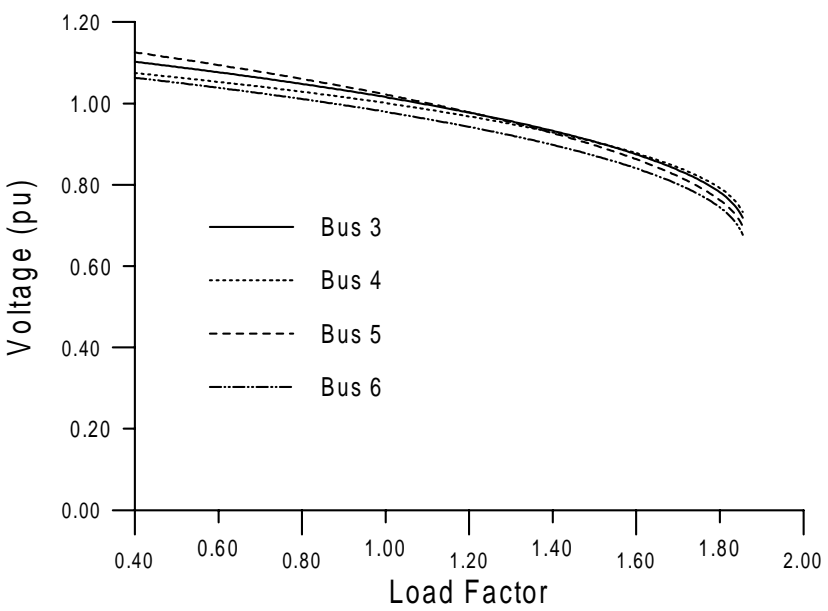

(b)

Fig. 3: Voltages of load buses

(a) Without optimization

(b) With Optimization

TABLE 3: LOAD FLOW RESULTS WITHOUT AND WITH OPTIMIZATION

\begin{tabular}{|c|c|c|c|c|c|}
\hline \multirow[t]{2}{*}{ Variable } & & \multicolumn{2}{|c|}{ Limits } & \multirow{2}{*}{$\begin{array}{c}\text { Without } \\
\text { Optimization }\end{array}$} & \multirow{2}{*}{$\begin{array}{c}\text { With } \\
\text { Optimization }\end{array}$} \\
\hline & & Low & High & & \\
\hline \multicolumn{6}{|l|}{ Control Variables } \\
\hline \multirow[t]{2}{*}{ Transformer Taps } & $t_{4}$ & 0.90 & 1.10 & 1.025 & 0.958 \\
\hline & $t_{7}$ & 0.90 & 1.10 & 1.100 & 0.984 \\
\hline \multirow[t]{2}{*}{ Generator Voltges (pu) } & $V_{l}$ & 1.00 & 1.10 & 1.050 & 1.092 \\
\hline & $V_{2}$ & 1.10 & 1.15 & 1.100 & 1.150 \\
\hline \multirow[t]{2}{*}{ Shunt Capacitors (MVAR) } & $Q_{c 4}$ & 0.00 & 5.00 & 0.000 & 5.000 \\
\hline & $Q_{c 6}$ & 0.00 & 5.50 & 0.000 & 5.500 \\
\hline \multicolumn{6}{|l|}{ Dependent Variables } \\
\hline \multirow[t]{2}{*}{ Generator MVAR } & $Q_{g 1}$ & -20.0 & 100.0 & 38.11 & 35.82 \\
\hline & $Q_{g 2}$ & -20.0 & 100.0 & 34.80 & 19.35 \\
\hline \multirow[t]{4}{*}{ Voltages at load Buses (pu) } & $V_{3}$ & 0.90 & 1.00 & 0.855 & 1.001 \\
\hline & $V_{4}$ & 0.90 & 1.00 & 0.953 & 1.001 \\
\hline & $V_{5}$ & 0.90 & 1.00 & 0.901 & 1.000 \\
\hline & $V_{6}$ & 0.90 & 1.00 & 0.933 & 0.984 \\
\hline System Losses (MW) & & --- & ---- & 11.61 & 8.880 \\
\hline
\end{tabular}


TABLE 4: BEHAVIOR OF L-INDEX WITH AND WITHOUT OPTIMIZATION

\begin{tabular}{ccc}
\hline $\begin{array}{c}\text { Bus } \\
\text { Number }\end{array}$ & $\begin{array}{c}\text { L-index Without } \\
\text { Optimization }\end{array}$ & $\begin{array}{c}\text { L-index With } \\
\text { Optimization }\end{array}$ \\
\hline 3 & 0.288 & 0.234 \\
4 & 0.211 & 0.178 \\
5 & 0.278 & 0.234 \\
6 & 0.258 & 0.218 \\
\hline
\end{tabular}

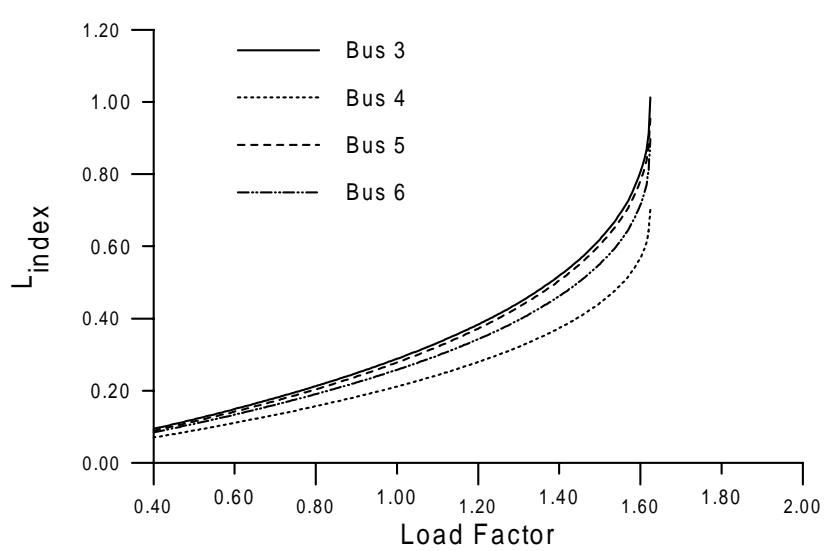

(a)

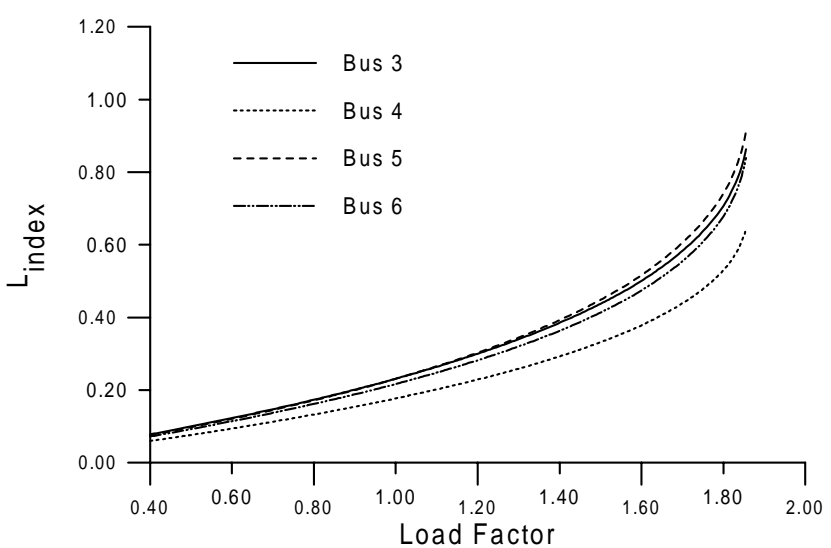

(b)

Fig. 4: L-index values at load buses
(a) Without optimization
(b) With Optimization

\section{CONCLUSIONS}

This paper has proposed an optimized voltage stability index using fast voltage stability indicator minimized by the simulated annealing optimization technique. The developed system has show accurate results, success in convergence to optimal solution. The results are obtained fast and direct. The conducted application on standard system has satisfactory results for optimal voltage stability level as well for extending the loading level of the system.

\section{ACKNOWLEDGEMENT}

The authors acknowledge the support of King Fahd University of Petroleum \& Mineral
[1] P..A. Lof, G. Andersson, and D..J. Hill, "Voltage Stability Indices For Stressed Power Systems," IEEE Trans. on Power Systems, Vol. 8, no. 1, Feb 1993, pp. 326-335.

[2] B. Gao, G. K. Morison, and P. Kundur, "Voltage Stability Evaluation Using Modal Analysis", IEEE Trans. on Power Systems, Vol. 7, no. 4, Nov 1992, pp. 1529-1542.

[3] N. Flatabo, R. Ognedal, and T. Carlsen," Voltage Stability Condition in a Power Transmission System Calculated by Sensitivity Methods", IEEE Trans. on Power Systems, Vol. 5, no. 4, Nov. 1990, pp. 1286-1293.

[4] M. M. Begovic and A. G. Phadke, "Control of Voltage Stability using Sensitivity Analysis", IEEE Trans. on Power Systems, Vol. 7, no. 1, Feb 1992, pp. 114-123.

[5] Y. Lin Chen, C. Wei Chang,etc, " Efficient Methods For Identifying Weak Nodes In Electrical Power Network, " IEE Proc. - Gener. Transm. Distrib. ,Vol 142, No. 3 , May 1995.

[6] K. Sakameto, Y. Tamura, etc, " Voltage Instability Proximity Index (VIPI) Based On Multiple Load Flow Solutions In Ill-conditioned Power Systems, " Proceeding of the 27th Conference on Decision and Control, Austin, Texas, December 1988.

[7] F. Gutina and B. Strmcnik, " Voltage Collapse Proximity Index Determination Using Voltage Phasors Approach," 94SM 510-8 PWRS, 1994.

[8] P. Kessel and H. Glavitsch, "Estimating The Voltage Stability of a Power System", IEEE Trans. on Power Delivery, Vol. PWRD-1, No 3, July 1986, pp. 346-354.

[9] C. Belhadj, R. Mohamedi, etc. "Voltage Stability Modelling and Real-Time Monitoring Using Expert System for Operation Assistance," IEEE Trans. on Power Systems, Vol. 11, No. 2, May 1996.

[10] R. C. Burchet, H. H. Happ, D. R. Vierath, and K. A. Wirgau, "Large Scale Optimal Power Flow," IEEE Trans., PAS-101, 1982, pp. 406-414.

[11] R. C. Burchet, H. H. Happ, and K. A. Wirgau, "Development in Optimal Power Flow," IEEE Trans., PAS101, 1982, pp. 3722-3732.

[12] K. R. C. Mamandur and R. D. chenoweth, "Optimal Control of Reactive Power Flow for Improvement in Voltage profiles and for Real Power Loss Minimization," IEEE Trans., PAS-100, 1981, pp. 3185-3194.

[13] R. R. Shoults and M. S. Chen, "Reactive Power Control by Least Squares Minimization," IEEE Trans., PAS-95, 1976, pp. 325-334.

[14] E. Hobson, "Network Constrained Reactive Power Control Using Linear Programming," IEEE Trans., PAS-99, 1980, pp. 868-887.

[15] Z. Wang, D. Peng, Q. Feng, H. Liu, and D. C. Yu, "A NonIncremental Model for Optimal Control of Reactive Power Flow," Electric Power Systems Research, Vol. 39, 1996, pp. 153-159.

[16] E. Aarts and J. Korst, Simulated Annealing and Boltzmann Machines: A Stochastic Approach to Combinatorial Optimization and Neural Computing, John Wiley \& Sons, 1989.

[17] N. Metropolis, A. Rosenbluth, M. Rosenbluth, A. Teller, and E. Teller, "Equation of State Calculations by Fast Computing Machines," Journal of Chemical Physics, Vol. 21, 1953, pp. 1087-1092. 